\title{
VAL1 as an assembly platform co-ordinating co-transcriptional repression and chromatin regulation at Arabidopsis FLC
}

\author{
Pawel Mikulski ${ }^{1,4}$, Philip Wolff ${ }^{1}$, Tiancong Lu ${ }^{1,2}$, Danling Zhu ${ }^{1,3} \&$ Caroline Dean ${ }^{1 *}$ \\ ${ }^{1}$ Cell and Developmental Biology, John Innes Centre, Norwich, UK \\ ${ }^{2,3,4}$ Present addresses: ${ }^{2}$ State Key Laboratory of Plant Genomics and National Center for Plant Gene \\ Research, Institute of Genetics and Developmental Biology, Chinese Academy of Sciences, Beijing, \\ China; ${ }^{3}$ SUSTech-PKU Institute of Plant and Food Science, Department of Biology, Southern \\ University of Science and Technology, Shenzhen, China; ${ }^{4}$ Department of Biochemistry, University of \\ Oxford, Oxford, UK
}

\begin{abstract}
Polycomb (PcG) silencing is crucial for development across eukaryotes, but how PcG targets are regulated is still incompletely understood. The slow timescale of cold-induced PcG silencing at Arabidopsis thaliana FLOWERING LOCUS C (FLC) makes it an excellent system to dissect this mechanism. Binding of the DNA binding protein VAL1 to an FLC intronic RY motif within the PcG nucleation region is an early step in the silencing process. VAL1 interacts with APOPTOSIS AND SPLICING ASSOCIATED PROTEIN (ASAP) complex and POLYCOMB REPRESSIVE COMPLEX 1 (PRC1). Here, we show that ASAP and PRC1 function as co-repressors that quantitatively regulate $F L C$ transcription. Upon the shift to cold PRC1-mediated H2Aub accumulates only at the nucleation region, is transiently maintained after transfer back to warm, but unlike the PRC2-delivered H3K27me3 does not spread across the locus. H2K27me3 thus provides long-term epigenetic silencing, whereas $\mathrm{H} 2 \mathrm{Aub}$ is a transient repression signal. Overall, our work highlights how a DNA sequence-specific binding protein can act as an assembly platform co-ordinating the cotranscriptional repression and chromatin regulation necessary for Polycomb silencing.
\end{abstract}

\section{Introduction}

From elegant genetic and molecular analysis in Drosophila the Polycomb group (PcG) proteins have been shown to play a central role in developmental gene regulation ${ }^{1}$. They function in distinct complexes to maintain epigenetic silencing of genomic targets ${ }^{2}$. The most well-studied complexes are POLYCOMB REPRESSIVE COMPLEX 1 (PRC1), which monoubiquitinates the core of histone H2A (H2Aub), and POLYCOMB REPRESSIVE COMPLEX 2 (PRC2), which methylates histone H3 tails at residue 27 (H3K27me). Initially thought to be sequentially recruited (PRC2, then PRC1) many studies have now demonstrated a much more complex scenario with variant and canonical PRC1 3,4 , interdependent recruitment of PRC1 and PRC2 and co-operative interactions mediating association to Polycomb Response Elements (PREs) ${ }^{5-8}$. There is also a lack of clarity about target site specificity as Polycomb complexes have been proposed to directly 'sample' transcriptionally permissive chromatin sites, rather than be recruited by developmental regulators 9 . Furthermore, the relationship between transcription and chromatin silencing appears to be able to switch between instructive and responsive ${ }^{10}$. Further dissection of the molecular events underpinning Polycomb silencing is therefore necessary to define the core mechanistic principles.

Arabidopsis FLOWERING LOCUS C (FLC) is a PcG target central to the process of vernalization ensuring flowering in favourable conditions ${ }^{11,12}$. FLC encodes a floral repressor whose expression is high in winter annual Arabidopsis accessions through the activity of FRIGIDA ${ }^{13,14}$. Short cold exposure in autumn/winter results in a relatively rapid transcriptional repression of $F L C$, in a process 
involving cold-induced $F L C$ antisense transcripts called COOLAIR ${ }^{15}$. Over the longer timescale of winter, $F L C$ is epigenetically silenced through a PRC2-dependent epigenetic switch ${ }^{16}$. This switch occurs at individual FLC alleles with a low probability ${ }^{17}$, leading to progressive silencing over the whole plant. A cold-induced step in this switch is the VIN3/VRN5-dependent nucleation of $\mathrm{H} 3 \mathrm{~K} 27 \mathrm{me} 3$ at an intragenic site that covers three nucleosomes between the transcription start site and the beginning of intron $1^{18-20}$. When plants return to warm temperatures, $\mathrm{H} 3 \mathrm{~K} 27 \mathrm{me} 3$ spreads to cover the entire gene, a feature required for the long-term epigenetic silencing throughout the rest of development ${ }^{17}$. The $F L C$ system thus provides the opportunity to dissect the components required for a PRC2 epigenetic switch.

Previously, we had shown that the transcriptional repressor VIVIPAROUS1/ABI3-LIKE (VAL1) was a central factor in the switching mechanism, necessary for H3K27me3 nucleation at $F L C^{21}$. VAL1 recognizes conserved RY motifs in FLC intron 1 with a one bp mutation in the first RY motif sufficient to completely block the vernalization process. The vall mutation attenuated FLC coldinduced transcriptional repression, but not the longer-term Polycomb memory. VAL1 was shown to interact with components of the APOPTOSIS AND SPLICING ASSOCIATED PROTEIN (ASAP), HDAC complex and PRC1, with components of ASAP interacting with the PRC2 accessory proteins VIN3 and VRN5. This then provided a connection between the sequence at the nucleation region and the machinery for long-term PcG silencing. However, the molecular role of the VAL1 interactors was not known.

Here, we investigate how VAL1 and its interactors ASAP and PRC1 function in FLC regulation. Overall, our results suggest that ASAP and PRC1 co-ordinate different steps in FLC regulation determining whether and how plants respond to cold, with the sequence-specific transcription factor VAL1 acting as the common recruitment platform. ASAP and PRC1 link co-transcriptional processing with chromatin regulation with $\mathrm{PRC} 1$-deposited $\mathrm{H} 2 \mathrm{AUb}$ accumulating at the nucleation region during cold exposure. However, this is only maintained at the nucleation region, unlike the PRC2-delivered H3K27me3 which spreads across the locus for long-term epigenetic silencing. Our work reveals how VAL1 functions as an assembly platform to co-ordinate co-transcriptional repression and chromatin regulation at Arabidopsis FLC.

\section{Results}

\section{Mutations of the ASAP complex affect FLC expression in warm but not cold-treated plants}

To analyse whether ASAP had a role in VAL1-dependent cold-induced FLC silencing we crossed asap mutants into a genotype carrying an active FRI allele in order to confer a vernalization requirement. The generated FRI asap genotypes were analysed for FLC expression over a vernalization time course. Plants were harvested before any cold exposure (non-vernalized; NV), immediately after 2,4 or 6 weeks of cold treatment (2WT0, 4WT0, 6WT0) and after 6 weeks of cold followed by a warm period for 7 days (6WT7). Interestingly, the major effect of the asap mutants in both FRI and fri genotypes was in warm (NV) conditions (Fig. 1a, b). The asap mutants did not influence the rate of FLC silencing after different cold and post-cold treatments (Fig. 1a). To understand the relationship of VAL1 and ASAP function we generated double mutant combinations in a Col-0 background and analysed in NV conditions. We observed a strong increase in FLC expression in warm-grown double mutants of vall asap (Fig. 1b), consistent with VAL1 interacting with many complexes to effect FLC silencing. We also found a stronger release of FLC silencing in combinations of asap mutants (Fig. 1b). As expected with the general correlation of sense and antisense transcription at $F L C^{22}$, COOLAIR was also upregulated in single asap mutants in warm conditions (Fig. S1a). These data suggest that a major role of ASAP at FLC is in quantitative 
transcriptional regulation that sets the level of FLC expression in the warm. Consistently, we found an interaction in yeast two hybrid between VAL1 and SIN3A-ASSOCIATED PROTEIN 18 (SAP18), (Fig. 1c,d, S1b), as similarly found in IP-MS with VAL1::VAL1-HA used as a bait ${ }^{21}$. This result is also matching the functional connection between SAP18 and VAL1 reported recently ${ }^{23}$. Given the physical interaction of ASAP components with the PRC2 accessory proteins VIN3 and VRN5, which mediate the cold-induced H3K27me3 nucleation ${ }^{21}$, the lack of an asap mutant phenotype during cold and post-cold steps likely indicates functional redundancy with other processes during the cold phase.

\section{SR45-dependent co-transcriptional repression at $F L C$}

A role for ASAP outside of Polycomb nucleation at FLC led us to analyse a link between cotranscriptional and chromatin regulation, as previously suggested for ASAP at other targets ${ }^{24}$. We selected the $s r 45$ mutant as an ASAP representative mutant with strong FLC upregulation (Fig. 1b) and profiled the co-transcriptional and chromatin changes at FLC. $s r 45$ increased relative $F L C$ transcript levels both in total (Fig, 1b) and chromatin-associated, nascent RNA (Fig. 1e) and COOLAIR increased co-ordinately (Fig. S1a). We did not observe changes in FLC splicing efficiency at introns 1-3 caused by loss of SR45 or other ASAP components (Fig. S2). However, sr45 caused higher levels of RNA PolII phosphorylated on serine-2 and serine-5 on its C-terminal domain (CTD) (Fig. 2a), which are associated with active transcription and increased the RNA PolII peak around the FLC nucleation region. Furthermore, the balance of active and repressive chromatin modifications was changed in warm grown sr45 plants, with increased H3K36me3 and decreased H3K27me3 at $F L C$ (Fig. 2b), in agreement with the transcriptional upregulation in the mutant. ASAP-dependent cotranscriptional regulation thus correlated with a variety of chromatin features at the FLC locus.

\section{VAL1 affects specific nucleosome dynamics}

A role for ASAP on co-transcriptional processes implied that its interactor, VAL1, may also have a similar function, especially given its impact on the active/repressive chromatin modifications ${ }^{21}$. To test this, we analysed chromatin-association of FLC RNA in warm-grown vall seedlings as a readout for bona fide $F L C$ transcription. Like the relative increase in total $\mathrm{RNA}^{21}$, and similar to $s r 45$ (Fig. 1e), vall increased chromatin associated FLC RNA (Fig. 3a). Given the VAL1 binding site is downstream of the $F L C$ promoter we asked if such transcriptional changes might be accompanied by changes in nucleosome dynamics ${ }^{25}$. We adapted the histone salt fractionation protocol that preferentially extracts nucleosomes recently disrupted by either transcription or remodelling ${ }^{25}$ and analysed FLC. The fraction of low-salt extractable nucleosomes decreased at the +1 and +2 nucleosomes in cold-treated samples (Fig. S3), agreeing with the previous observation of coldinduced nucleosome stabilization ${ }^{19}$. Furthermore, we observed that vall increased the low-salt extractable fraction for nucleosomes +1 and +3 , but not for nucleosome +2 (Fig. 3b, c). Nucleosomes +1 and +3 correspond to the edges of the $\mathrm{H} 3 \mathrm{~K} 27$ me3-enriched nucleation region found in FLC after cold exposure ${ }^{26}$. These VAL1-dependent changes were confined to chromatin extracted from warmgrown vall seedings and were not found in cold-treated samples (Fig. S3). Interestingly, they were not connected to gross changes in chromatin accessibility as judged by FAIRE (formaldehyde-assisted isolation of regulatory elements, a technique using nuclease hypersensitivity to identify open and therefore potentially regulatory chromatin) (Fig. 3d). Overall, these data connect VAL1-induced cotranscriptional quantitative regulation to nucleosome dynamics at the edges of the FLC nucleation region, a region where RNA PolII occupancy is high.

H2Aub accumulates during cold at the FLC nucleation region but does not spread like H3K27me3 upon return to warm 
144 To begin to investigate the functional significance of the VAL1-PRC1 interaction we undertook analysis of $\mathrm{H} 2 \mathrm{AUb}$ accumulation at $F L C$ on the vernalization-sensitive genotype ColFRI after different cold exposure. Reduced $F L C$ expression correlated with increasing H2Aub levels as plants were exposed to progressive cold (Fig. 4a). H2Aub accumulated at the FLC nucleation region with dynamics similar to PRC2-induced H3K27me3 nucleation. To establish whether the H2Aub correlated with $F L C$ transcriptional activity we then analysed lines with or without FRIGIDA (FRI), which is the main determinant distinguishing vernalization requirement or rapid-cycling habit. In the rapid-cycling Col-0, H2Aub levels at FLC were much higher than in Col-FRI reflecting the $\sim 20 \mathrm{x}$ reduction in FLC transcription (Fig. 4b). These results suggest that $\mathrm{H} 2 \mathrm{Aub}$ is inversely correlated with transcription and PRC1 has a typical transcription repression function at $F L C$, rather than a contextdependent role in transcriptional activation ${ }^{3,4}$.

We then tested how H2Aub changes in warm conditions post-cold, when FLC chromatin transitions into a stably repressed state. We measured $\mathrm{H} 2 \mathrm{Aub}$ and $\mathrm{H} 3 \mathrm{~K} 27 \mathrm{me} 3$ at $F L C$ during the vernalization timecourse ${ }^{26}$. As expected, H3K27me3 accumulated in the nucleation region during cold exposure $(6 \mathrm{~W}+$ day 0$)$ and spread across the whole gene body after transfer back to warm $(6 \mathrm{~W}+$ day $10 / 14 / 20)$ (Fig. 4c). H2Aub accumulated in the nucleation region during cold, similar to the H3K27me3 dynamics, and continued to increase for 10 and 14 days after transfer back to the warm but did not spread across the locus (Fig. 4c). Interestingly, 20 days after transfer back to the warm, the H2Aub peak at the nucleation decreased back to levels seen at the end of $6 \mathrm{~W}$ cold. These dynamics closely match the occupancy of the VRN5 accessory protein at the nucleation region ${ }^{17}$.

\section{VAL1, PRC1 and NDX physically interact and are required for H2Aub accumulation at the} $F L C$ nucleation region

168 Genetic dissection of the role of $\mathrm{H} 2 \mathrm{AUb}$ at $F L C$ is complex as the multiple Arabidopsis PRC1 components are functionally redundant and single PRC1 mutants do not show a changed FLC expression relative to wildtype ${ }^{27,28}$ (Fig. 5a). Loss of all three BMI subunits (A, B, C) de-represses $F L C$ expression in warm-grown Col-0 plants up to 25 fold $^{29}$. Higher order PRC1 mutant combinations are seedling lethal or show severe developmental aberrations ${ }^{27,30,31}$. In addition, Arabidopsis PRC1 has a range of partners central to its involvement in different developmental pathways ${ }^{32}$. However, to pursue the consequence of the VAL1-PRC1 interaction we analysed $F L C$ expression differences in vall bmilB line. vall bmilB did not increase $F L C$ expression above vall alone in any condition tested (Fig. 5b, c) or further influence $F L C$ H2Aub levels in warm-grown plants (Fig. 5d). In addition, vall bmilB did not show FLC/COOLAIR reactivation post-cold (Fig. S4). This was also true for the combination vall ringlA (Fig. S5). We did find an additive effect of the vall bmilB combination on COOLAIR expression (Fig. S6), so for this aspect of FLC regulation other PRC1 components do not completely cover loss of BMI1B function.

We undertook further IP-MS experiments with VAL1-HA as a bait and found a robust interaction between VAL1 with other proteins (Table S1), including PRC1 members, as well as NODULIN HOMEOBOX (NDX) (Fig. 6a). Interestingly, NDX has been previously implicated in single-stranded DNA recognition and stabilization of the R-loop at $F L C^{33}$. Using yeast-two-hybrid analysis we found that NDX does not interact directly with VAL1 but instead interacts with RING1A and RING1B (PRC1 components) (Fig. 6b, c, S7), as also found recently ${ }^{34}$. Genetic analysis between vall, prc1 mutants and $n d x l$ revealed that NDX is required for the FLC upregulation caused by vall, and loss of PRC1 does not affect the consequences of NDX deficiency (Fig. 5a); COOLAIR was upregulated recognized RY motifs are necessary for full H2Aub enrichment at FLC, as well as H3K27me3 (Figs. 
$1926 \mathrm{~d}$, e, S9 and as published $\mathrm{in}^{21}$ ). NDX has been shown to associate with the 3' region of $F L C$ and stabilize R-loops ${ }^{33,35}$, so it will be important to establish if these genetic interactions point to an additional NDX/R-loop interaction in the $F L C$ nucleation region or are the result of the gene loop ${ }^{36}$.

H2Aub enrichment at FLC was unaffected in mutants of the core PRC2 component VERNALIZATION 2, or the PRC2-accessory protein VERNALIZATION 5 in non-vernalized conditions but was slightly reduced after 6 weeks cold exposure (Fig. S10). The lack of difference in NV conditions is in agreement with H2Aub being generally independent of LHP1 and PRC2 function in Arabidopsis, as previously reported ${ }^{37}$. However, the small effect after cold potentially connects close functioning of VAL1, NDX and PRC1 in FLC co-transcriptional repression to PRC2 nucleation and chromatin silencing.

\section{Discussion}

How transcriptional repression links to epigenetic silencing is far from understood. Cell type-specific or developmentally induced transcriptional repressors bind different Polycomb complexes but direct recruitment models are too simplistic ${ }^{9,10}$; linear genetic hierarchies have not been established and functional output of PREs has been shown to be heavily dependent on the chromatin context ${ }^{38}$. Our findings that VAL1 acts as an assembly platform co-ordinating ASAP and PRC1 activities that occur at different stages in the FLC regulation helps explain some of this complexity. It also raises interesting parallels to the non-linear recruitment activities of the PcG proteins at Drosophila PREs $^{7,39,40}$, with ASAP co-transcriptional repressors and PRC1 components likely redundantly establishing the chromatin environment required for robust PRC2 silencing (Fig. 7).

ASAP and PRC1 have been documented to have very different roles in gene regulation in different organisms. ASAP has previously been shown to play a role as part of the spliceosome and exon junction complexes in many organisms ${ }^{24,41}$, whereas, PRC1 functionality is associated with $\mathrm{H} 2 \mathrm{AUb}$ and transcriptional silencing ${ }^{42}$. In plants, ASAP has been associated with regulation of innate immunity $^{43}$ and abiotic responses ${ }^{44}$ and considered to function through altering RNA metabolism or alternative splicing ${ }^{45,46}$. However, we did not detect any effect of a sr45 mutation on alterative splicing or splicing efficiency of the FLC sense strand introns. Instead, we found changes linked to transcriptional repression - RNA PolII post-translational modifications and classic histone modifications associated with Polycomb target ON or OFF states. We thus propose that SR45, as an ASAP component, influences a co-transcriptional step that may function to influence RNA PolII complex composition and thus the chromatin modifications deposited during transcription. Since there is physical linkage of VIN3 and VRN5 ${ }^{21}$ to ASAP components, the ASAP complex is likely to function at $F L C$ both during growth in the warm, as judged by mutant phenotypes, and redundantly with other functions in FLC silencing during cold exposure. Changed co-transcriptional regulation with RNA PolII both involved in histone modification deposition, but also sensitive to histone modifications on the chromatin template, would seem a probable mechanism underlying the instructive/responsive transcription-chromatin relationship.

The PRC1-mediated H2AUb modification has been associated with transcriptional regulation and epigenetic stability in Drosophila and mammalian cells ${ }^{42}$. At $F L C$, the PRC1-H2AUb is associated with transcriptional repression, either in low FLC expressing genotypes (Col-0) in warm conditions, or in response to cold in the high FLC expressing Col-FRI genotype. In this respect it appears to function at $F L C$ more as a variant PRC1 rather than a canonical PRC1. The timescale of the VAL1PRC1-mediated $\mathrm{H} 2 \mathrm{Aub}$ accumulation at the $F L C$ nucleation region during cold exposure suggests an intermediary role between the transcriptional silencing determined by ASAP-VAL1 and the long-term 
240 PRC2 epigenetically stable spread state. However, the genetic redundancy makes PRC1 functionality

241 hard to tease apart so we were unable to dissect the interdependency of PRC1-delivered $\mathrm{H} 2 \mathrm{AUb}$ and PRC2-delivered H3K27me3 in the epigenetic switch at FLC. Therefore, how VAL1 interaction with ASAP and PRC1 is co-ordinated spatially and temporally at the $F L C$ nucleation region remains to be fully elaborated.

VAL1 interacts with ASAP via SAP18 and PRC1 but has also been shown to interact with LHP1 ${ }^{32}$, histone deacetylases (HDA9, HDA6) ${ }^{47,48}$ and Mediator complex component (MED13) ${ }^{47}$. It will be important in the future to understand how one protein can mediate all these interactions and whether they are reinforced through multivalent interactions with other proteins at the nucleation region, like the Drosophila PRE situation. The original intronic mutation in the RY motif caused relatively little warm mis-regulation of $F L C^{21}$, just attenuation of the cold-induced transcriptional silencing without a subsequent effect on stability of the silenced state. The stronger phenotype of the VAL1 interactors in the warm argues that they also function independently of VAL1. The lack of phenotype in the cold suggests that functionally redundant, cold-specific regulators are still to be identified. These are likely to involve the antisense transcripts at FLC (COOLAIR) that contribute to transcriptional silencing during cold exposure, which associate with the chromatin at the 5' end of $F L C$, and in which natural variation has a functional consequence ${ }^{49,50}$.

Overall, we interpret our data as showing that VAL1 functions as an assembly platform to co-ordinate co-transcriptional repression and chromatin regulation at Arabidopsis FLC. We envisage that transcription is downregulated by multiple repressor complexes (ASAP and PRC1) either linked to RNA PolII and interacting with VAL1 at the nucleation region or assembled at the nucleation region. These appear to function independently in the warm, with H2AUb playing a major role in the cold associated with FLC silencing, alongside PRC2 deposition of H3K27me3. Given the parallels with the Drosophila PcG mechanism the activities of these many protein complexes are likely to function cooperatively to influence the different steps in the mechanism. Further elucidation will require detailed analysis of the co-transcriptional silencing, chromatin modification and nucleosome remodelling.

\section{Material and methods}

\section{Plant material}

272 All mutants and transgenic lines were either in Col-0 or $\mathrm{FRI}^{\mathrm{sf} 2}$ background ${ }^{21,50}$. Unless otherwise

273 stated, experiments in non-vernalized conditions used Col-0 as a wildtype. The analyses in 274 vernalization timecourse used Col FRI ${ }^{\mathrm{s} 2}$ (Col FRI) as a wildtype to capture dynamics of cold-induced $275 \quad F L C$ expression and chromatin changes. Mutant alleles were used as described previously: bmilB 276 (drip1-1), bmilA (drip2-1), ring1A (GK-293A04), ring1B (SALK_117958), ndx1-4, flc (flc-2), RY 277 motif (vrn8), val1-2, vin3-4, vrn2-1, vrn5-8, sap18 (SALK_02363C), sr45 (SALK_018273), acinus 278 (SALK_078554) ${ }^{17,21,33,34,51}$. Double mutants were generated by crosses between homozygous single mutant lines and selected by PCR-based genotyping using primers listed in Table S2.

\section{Growth conditions}

Seeds were surface sterilized and sown on MS-GLU (MS without glucose) media plates and stratified at $4^{\circ} \mathrm{C}$ in the dark for 2 days. For non-vernalized (NV) conditions, seedlings were grown for 14 days in long-day conditions ( $16 \mathrm{~h}$ light, $8 \mathrm{~h}$ darkness with constant $20^{\circ} \mathrm{C}$ ). For vernalization treatment, seedlings were grown at $5^{\circ} \mathrm{C}$ in short-day conditions $\left(8 \mathrm{~h} \mathrm{light}, 16 \mathrm{~h}\right.$ darkness with constant $\left.5^{\circ} \mathrm{C}\right)$ for number of weeks indicated in the respective experiment description. 
$2874 \mathrm{~g}$ of Arabidopsis seedlings were crosslinked and ground in fine powder by liquid $\mathrm{N} 2$, following 288 ChIP protocol ${ }^{21}$. Immunoprecipitation of protein complexes was done as described in ${ }^{17}$ using Protein 289 A beads (Thermo Fisher Scientific, \#10008D) coupled with HA antibody (CST, \#3724). IP product 290 was resuspended with 2X NuPAGE sample buffer (Thermo Fisher Scientific, \#NP0007) and 291 denatured at $70^{\circ} \mathrm{C}$ for $15 \mathrm{~min}$ with $100 \mathrm{mM}$ DTT. The final samples were run on NuPAGE Bis-Tris 292 gels (Thermo Fisher Scientific, \# NP0321) and subjected to in-gel digestion.

293 Protein bands corresponding to heavy and light chains of antibody were excised from NuPAGE gels.

294 The rest of gels were combined and performed in-gel digestion as described previously ${ }^{52}$. Briefly, gel 295 pieces were destained and then incubated with $10 \mathrm{ng} / \mu \mathrm{l}$ trypsin (Promega, \#V5111) overnight at $37^{\circ} \mathrm{C}$. 296 Peptides were extracted by incubation with 5\% trifluoroacetic acid (TFA) for $1 \mathrm{~h}$, followed by 297 addition of $2.5 \% \mathrm{TFA} / 50 \%$ acetonitrile $(\mathrm{ACN})$ for $1 \mathrm{~h}$ at $37^{\circ} \mathrm{C}$. The combined supernatants were dried 298 in a SpeedVac concentrator (Thermo Fisher Scientific, \#SPD120) for mass spectrometry (MS) 299 analysis.

300 Mass spectrometry

301 Samples were analyzed by LC-MS using UltiMate ${ }^{\mathrm{TM}} 3000$ RSLCnano System (Thermo Fisher 302 Scientific, \#ULTIM3000RSLCNANO) interfaced with Q Exactive HF. Digested samples were loaded 303 on a fused silica trap column Acclaim PepMap 100, $0.075 \mathrm{~mm}$ x $2 \mathrm{~cm}$ (Thermo Fisher Scientific, 304 \#164569). After washing for $5 \mathrm{~min}$ at $5 \mu \mathrm{l} / \mathrm{min}$ with $0.1 \%$ TFA, the trap column was brought in-line 305 with an analytical column (Nanoease MZ peptide BEH C18, 130A, 1.7um, $0.075 \mathrm{~mm}$ x250 mm, 306 Waters, \# 186008800) for LC-MS/MS. Peptides were fractionated at $300 \mathrm{~nL} / \mathrm{min}$ using a segmented 307 linear gradient from 4 to $90 \%$ B (A: $0.1 \%$ formic acid, B: $0.08 \%$ formic acid, $80 \%$ ACN): 5 min, $4-$ $30810 \% \mathrm{~B} ; 60 \mathrm{~min}, 10-40 \% \mathrm{~B} ; 15 \mathrm{~min}, 40-55 \% \mathrm{~B} ; 10 \mathrm{~min}, 55-90 \% \mathrm{~B}$. Mass spectrometry data was 309 acquired using a data-dependent acquisition procedure with a cyclic series of a full scan acquired with 310 a resolution of 120,000 followed by MS/MS scans (30\% of collision energy in the HCD cell) with 311 resolution of 30,000 of 20 most intense ions with dynamic exclusion duration of $20 \mathrm{sec}$.

312 The peak list of the LC-MS/MS were generated by Thermo Proteome Discoverer (v. 2.1) into 313 MASCOT Generic Format (MGF) and searched against TAIR protein database (containing 35,386 314 sequences) using an in-house version of X! tandem (SLEDGEHAMMER (2015.09.01), the gpm.org) 315 with carbamidomethylation on cysteine as fixed modification and oxidation of methionine as variable 316 modification. A $+/-7 \mathrm{ppm}$ and $20 \mathrm{ppm}$ were used as tolerance for precursor and product ions 317 respectively. Trypsin/P was selected as the digestion enzyme and one missed cleavage per peptide 318 was allowed. The False Discovery Rate was estimated for all samples by using a reverse database 319 (FDR <0.01). All identified spectra belong to Arabidopsis database and peptide $\log (\mathrm{E})<=-2(\mathrm{E}$ 320 value $<=0.01$ ) was reported. Spectral counts of the proteins in each sample were used for relative 321 quantitation.

\section{Yeast two hybrid analysis}

323 Yeast two hybrid was performed as described elsewhere ${ }^{53}$. Yeast cultures were grown at $28^{\circ} \mathrm{C}$ on

324 Yeast Peptone Dextrose (YPD) or on selective Synthetic Dextrose (SD) media. The AH109 strain was 325 used for transformation, following protocol described in the Yeast Protocols Handbook (version no. 326 PR973283 21; Clontech), with both Gal4-BD and Gal4-AD constructs added. Transformants were selected on SD medium lacking Trp and Leu (SD-LW). Transformed yeast were grown to OD600 and spotted onto selective SD medium additionally lacking His (SD-LWH) which permitted the identification of weak protein interactions. Yeast dilutions during spotting were: 1/1, 1/5, 1/25, 1/25.

331 Total RNA extraction was performed using the hot phenol method, as described elsewhere ${ }^{54}$. 332 Genomic DNA contamination was removed using TURBO DNA-free kit (Invitrogen, \#AM1907) following the manufacturer's guidelines. cDNA was synthesised using SuperScript IV First-strand 334 Synthesis System (Invitrogen, \#18091050), using gene-specific primers. cDNA was diluted 10 times 
before qPCR. All primers are listed in the Table S2. cDNA was amplified using SYBR Green I Master (Roche, 04887352001) and run on LightCycler 480 machine (Roche, 05015243001). Ct values were normalized to geometric mean of reference genes: UBIQUITIN CARRIER PROTEIN 1 (UBC) and/or SERINE/THREONINE PROTEIN PHOSPHATASE 2A (PP2A). Chromatin-associated RNA has been isolated using urea as published before ${ }^{55}$.

\section{$340 \quad$ Nucleosome stability analysis}

341 The protocol has been adapted from ${ }^{56} .2 \mathrm{~g}$ Arabidopsis seedlings were ground in liquid nitrogen, resuspended in $35 \mathrm{ml}$ NIB buffer $(10 \mathrm{mM}$ MES-KOH pH=5.4, $10 \mathrm{mM} \mathrm{NaCl}, 10 \mathrm{mM} \mathrm{KCl}, 2.5 \mathrm{mM}$ EDTA, $250 \mathrm{mM}$ Sucrose, $0.1 \mathrm{mM}$ Spermine, $0.5 \mathrm{mM}$ Spermidine, 1mM DTT, 1x Protease inhibitor cocktail EDTA-free (Thermo Fisher Scientific, \# A32965)) and filtered through one layer of miracloth. $20 \%$ Triton X-100 was added to final concentration of $0.35 \%$, the extract was rotated at $4^{\circ} \mathrm{C}$ for $10 \mathrm{~min}$ and spun at $1000 \mathrm{~g}$ for $10 \mathrm{~min}$. The pellet was transferred to Low Protein Binding tubes (Thermo Fisher Scientific, \#90410) and washed twice with MNase buffer (10 mM Tris-HCl $\mathrm{pH}=8,5 \mathrm{mM} \mathrm{NaCl}, 2.5 \mathrm{mM} \mathrm{CaCl} 2,2 \mathrm{mM} \mathrm{MgCl} 2$, 1x Protease inhibitor cocktail EDTA-free (Thermo Fisher Scientific, \# A32965))) at 1000g for $3 \mathrm{~min}$. The pellet was weighted and MNase buffer was added in ratio $5 \mu \mathrm{L}$ buffer: $1 \mu \mathrm{g}$ pellet. The mixture was pre-warmed at $37^{\circ} \mathrm{C}$ for $5 \mathrm{~min} .150 \mu \mathrm{L}$ of the mixture was put aside as an uncut sample and other $150 \mu \mathrm{L}$ was subjected to $5 \mathrm{U} / \mathrm{ml}$ (final concentration) MNase (Takara Bio, \#2910A) enzymatic digestion at $37^{\circ} \mathrm{C}$ for $10 \mathrm{~min}$. The concentration of MNase was experimentally tested to obtain nucleosome ladder as $i^{47}$. The reaction was stopped by adding EGTA to final concentration of $2 \mathrm{mM}$. The mixture was spun at $1000 \mathrm{~g}$ for 3 min, supernatant was removed, the pellet resuspened in $80 \mathrm{mM}$ salt buffer $(80 \mathrm{mM} \mathrm{NaCl})$ and rotated at $4^{\circ} \mathrm{C}$ for $15 \mathrm{~min}$. The samples were spun at $1000 \mathrm{~g}$ for $3 \mathrm{~min}$ and the supernatant was collected as mobile nucleosome fraction. Samples were adjusted to $500 \mu \mathrm{L}$ and subjected to DNA recovery by adding $1 / 50$ volume $0.5 \mathrm{M}$ EDTA, $1 / 50$ vol. $5 \mathrm{M} \mathrm{NaCl}$ and $2 \mu \mathrm{L}$ RNAse A (Thermo Fisher Scientific, \#EN0531). The samples were incubated at $37^{\circ} \mathrm{C}$ for $30 \mathrm{~min}$. Proteins were removed by adding 1/16 vol. 10\% SDS to final concentration $0.63 \%$ and $2.5 \mu \mathrm{L}$ Proteinase K (Ambion, \#AM2546), followed by incubation at $50^{\circ} \mathrm{C}$ for $1 \mathrm{~h}$. The samples were then subjected to standard phenol-chloroformisoamyl alcohol and DNA precipitation with ethanol. The enrichment was calculated by qPCR with primers indicated in Table S2. Ct values in mobile nucleosome fraction were normalized to uncut DNA and Gypsy-like transposon (At4g07700) as an internal control, following normalizations for published in ${ }^{57}$.

\section{Micrococcal nuclease assay (MNase-qPCR)}

$2 \mathrm{~g}$ Arabidopsis seedlings were ground in liquid nitrogen and the pellet was resuspended in Honda buffer ${ }^{21}$, filtered through miracloth and spun at $1000 \mathrm{~g}, 4^{\circ} \mathrm{C}$ for $10 \mathrm{~min}$, similarly to ChIP protocol. Subsequently, a pellet was washed twice in MNase buffer $(10 \mathrm{mM}$ Tris- $\mathrm{HCl} \mathrm{pH}=8,5 \mathrm{mM} \mathrm{NaCl}, 2.5$ $\mathrm{mM} \mathrm{CaCl} 2,2 \mathrm{mM} \mathrm{MgCl} 2,1 \mathrm{x}$ Protease inhibitor cocktail EDTA-free (Thermo Fisher Scientific, \# A32965))) and finally resuspended in $1 \mathrm{~mL}$ MNase buffer. The samples were divided into $300 \mu \mathrm{L}$ uncut control and $300 \mu \mathrm{L}$ MNase-digested sample. The mixtures were prewarmed at $37^{\circ} \mathrm{C}$ for $5 \mathrm{~min}$ and digested samples were treated with $66.6 \mathrm{U} / \mathrm{mL}$ (final conc.) MNase enzyme (Takara Bio, $\# 2910 \mathrm{~A}$ ) at $37^{\circ} \mathrm{C}$ for $30 \mathrm{~min}$. The reactions were stopped by adding $1 / 100$ volume $0.5 \mathrm{M}$ EDTA and subjected to protein extraction by adding $0.05 \%$ (final conc.) SDS, followed by standard phenolchloroform-isoamyl alcohol and DNA precipitation with isopropanol. The enrichment was calculated by QPCR with primers indicated in Table S2. Ct values in mobile nucleosome fraction were normalized to uncut DNA.

\section{Formaldehyde-assisted isolation of regulatory elements (FAIRE)}

380 FAIRE was performed as shown in $^{57}$. The enrichment was assessed by qPCR using primers indicated 
crosslinked sample (FAIRE) to non-crosslinked sample (UN-FAIRE) and then to UBC9

(AT4G27960) as an internal control.

\section{Chromatin immunoprecipitation}

Histone modification and PolII ChIP was performed as previously described ${ }^{17,55}$, respectively. Immunoprecipitation was done with antibodies: anti-H3 (Abcam, \#ab1791), anti-H3K27me3 (Millipore, \#07-449), H2AK119ub (Cell Signalling Technology, \#8240), anti-H3K36me3 (Abcam, \#ab9050). PolII antibodies were as in ${ }^{58}$. All ChIP experiments were followed by qPCR with indicated primer pairs (Table S2).

\section{Statistical analysis}

Statistical analyses were performed in Microsoft Excel and R Studio (R v4.0.2). P-value, p-value levels and sample number are included in figures and figure legends. Significance calculations with Student T-test were precluded by F-test to determine the usage of heteroscedastic or homoscedastic test. Unless otherwise stated, Student T-tests were two-tail. P-value levels were marked by asterisks on the figures as follows: $*(\mathrm{p} \leq 0.05), * *(\mathrm{p} \leq 0.01), * * *(\mathrm{p} \leq 0.001)$.

\section{References}

1. Schuettengruber, B., Bourbon, H. M., Di Croce, L. \& Cavalli, G. Genome Regulation by Polycomb and Trithorax: 70 Years and Counting. Cell 171, 34-57 (2017).

2. Di Croce, L., Helin, K. Transcriptional regulation by Polycomb group proteins. Nat Struct Mol Biol 20, 1147-1155 (2013).

3. Fursova, N. A. et al. Synergy between Variant PRC1 Complexes Defines Polycomb-Mediated Gene Repression. Mol. Cell 74, 1020-1036.e8 (2019).

4. Scelfo, A. et al. Functional Landscape of PCGF Proteins Reveals Both RING1A/BDependent-and RING1A/B-Independent-Specific Activities. Mol. Cell 74, 1037-1052.e7 (2019).

5. Kahn, T. G. et al. Interdependence of PRC1 and PRC2 for recruitment to Polycomb Response Elements. Nucleic Acids Res. 44, 10132-10149 (2016).

6. Kahn, T. G., Stenberg, P., Pirrotta, V. \& Schwartz, Y. B. Combinatorial Interactions Are Required for the Efficient Recruitment of Pho Repressive Complex (PhoRC) to Polycomb Response Elements. PLoS Genet. 10, e1004495 (2014).

7. Alfieri, C. et al. Structural basis for targeting the chromatin repressor Sfmbt to Polycomb response elements. Genes Dev. 27, 2367-2379 (2013).

8. Frey, F. et al. Molecular basis of PRC1 targeting to polycomb response elements by PhoRC. Genes Dev. 30, 1116-1127 (2016).

9. Klose, R. J., Cooper, S., Farcas, A. M., Blackledge, N. P. \& Brockdorff, N. Chromatin Sampling - An Emerging Perspective on Targeting Polycomb Repressor Proteins. PLoS Genet. 9, e1003717 (2013).

10. Berry, S., Dean, C. \& Howard, M. Slow Chromatin Dynamics Allow Polycomb Target Genes to Filter Fluctuations in Transcription Factor Activity. Cell Syst. 4, 445-457.e8 (2017).

11. Michaels, S. D. \& Amasino, R. M. FLOWERING LOCUS C encodes a novel MADS domain protein that acts as a repressor of flowering. Plant Cell 11, 949-956 (1999).

12. Sheldon, C. C. The molecular basis of vernalization: The central role of FLOWERING LOCUS C (FLC). Proc. Natl. Acad. Sci. 97, 3753-3758 (2000).

13. Shindo, C. et al. Role of FRIGIDA and FLOWERING LOCUS C in determining variation in flowering time of Arabidopsis. Plant Physiol. 138, 1163-1173 (2005).

14. Johanson, U. et al. Molecular analysis of FRIGIDA, a major determinant of natural variation in Arabidopsis flowering time. Science 290, 344-347 (2000).

15. Swiezewski, S., Liu, F., Magusin, A. \& Dean, C. Cold-induced silencing by long antisense transcripts of an Arabidopsis Polycomb target. Nature 462, 799-802 (2009).

16. Berry S., Hartley M., Olssen TS, Dean C. Howard M. Local chromatin environment of a Polycomb target instructs its own epigenetic inheritance. eLife 4, e07205 (2015).

17. Yang, H. et al. Distinct phases of Polycomb silencing to hold epigenetic memory of cold in 
Arabidopsis. Science. 357, 1142-1145 (2017).

18. De Lucia, F., Crevillen, P., Jones, A. M. E., Greb, T. \& Dean, C. A PHD-Polycomb Repressive Complex 2 triggers the epigenetic silencing of FLC during vernalization. Proc. Natl. Acad. Sci. U. S. A. 105, 16831-16836 (2008).

19. Finnegan, JE. Time-dependent stabilization of the +1 nucleosome is an early step in the transition to stable cold-induced repression of FLC. Plant J. 84, 875-885 (2015).

20. Wood, C. C. et al. The Arabidopsis thaliana vernalization response requires a Polycomb-like protein complex that also includes VERNALIZATION INSENSITIVE 3. Proc. Natl. Acad. Sci. U. S. A. 103, 14631-14636 (2006).

21. Quiesta, J. I. et al. Arabidopsis transcriptional repressor VAL1 triggers Polycomb silencing at FLC during vernalization. Science 353, 485-8 (2016).

22. Marquardt, S. et al. Functional Consequences of Splicing of the Antisense Transcript COOLAIR on FLC Transcription. Mol. Cell 54, 156-165 (2014).

23. Baile, F., Merini, W., Hidalgo, I. \& Calonje, M. EAR domain-containing transcription factors trigger PRC2-mediated chromatin marking in Arabidopsis. Plant Cell, koab139 (2021).

24. Deka, B. \& Singh, K. K. Multifaceted regulation of gene expression by the Apoptosis- and Splicing-Associated Protein complex and its components. Int. J. Biol. Sci. 13, 545-560 (2017).

25. Henikoff, S., Henikoff, J. G., Sakai, A., Loeb, G. B. \& Ahmad, K. Genome-wide profiling of salt fractions maps physical properties of chromatin. Genome Res. 19, 460-469 (2009).

26. Yang, H., Howard, M. \& Dean, C. Antagonistic roles for H3K36me3 and H3K27me3 in the cold-induced epigenetic switch at Arabidopsis FLC. Curr. Biol. 24, 1793-1797 (2014).

27. Xu, L. \& Shen, W. H. Polycomb Silencing of KNOX Genes Confines Shoot Stem Cell Niches in Arabidopsis. Curr. Biol. 18, 1966-1971 (2008).

28. Chen, D., Molitor, A., Liu, C. \& Shen, W. H. The Arabidopsis PRC1-like RING-finger proteins are necessary for repression of embryonic traits during vegetative growth. Cell Res. 20, 1332-1344 (2010).

29. Merini, W. et al. The Arabidopsis Polycomb Repressive Complex 1 (PRC1) components AtBMI1A, B, and C impact gene networks throughout all stages of plant development. Plant Physiol. 173, 627-641 (2017).

30. Bratzel, F., López-Torrejón, G., Koch, M., Del Pozo, J. C. \& Calonje, M. Keeping cell identity in arabidopsis requires PRC1 RING-finger homologs that catalyze H2A monoubiquitination. Curr. Biol. 20, 1853-1859 (2010).

31. Yang, C. et al. VAL-and AtBMI1-Mediated H2Aub initiate the switch from embryonic to postgerminative growth in Arabidopsis. Curr. Biol. 23, 1324-1329 (2013).

32. Yuan, L. et al. The transcriptional repressors VAL1 and VAL2 recruit PRC2 for genome-wide Polycomb silencing in Arabidopsis. Nucleic Acids Res. 49, 98-113 (2021).

33. Sun, Q., Csorba, T., Skourti-Stathaki, K., Proudfoot, N. J. \& Dean, C. R-loop stabilization represses antisense transcription at the Arabidopsis FLC locus. Science 340, 619-621 (2013).

34. Zhu, Y. et al. The Arabidopsis Nodulin Homeobox Factor AtNDX interacts with AtRING1A/B and negatively regulates abscisic acid signaling. Plant Cell 32, 703-721 (2020).

35. Xu, C. et al. R-loop resolution promotes co-transcriptional chromatin silencing. Nat. Commun. 12, 1790 (2021).

36. Crevillén, P., Sonmez, C., Wu, Z. \& Dean, C. A gene loop containing the floral repressor FLC is disrupted in the early phase of vernalization. EMBO J. 32, 140-148 (2013).

37. Zhou, Y., Romero-Campero, F. J., Gómez-Zambrano, Á., Turck, F. \& Calonje, M. H2A monoubiquitination in Arabidopsis thaliana is generally independent of LHP1 and PRC2 activity. Genome Biol. 18, 69 (2017).

38. De, S., Cheng, Y., Sun, M. A., Gehred, N. D. \& Kassis, J. A. Structure and function of an ectopic Polycomb chromatin domain. Sci Adv. 5, eaau9739 (2019)

39. Sengupta, A. K., Kuhrs, A. \& Müller, J. General transcriptional silencing by a Polycomb response element in Drosophila. Development 131, 1959-1965 (2004).

40. Christen, B. \& Bienz, M. Imaginal disc silencers from Ultrabithorax: evidence for Polycomb response elements. Mech. Dev. 48, 255-266 (1994).

41. Schwerk, C. et al. ASAP, a Novel Protein Complex Involved in RNA Processing and Apoptosis. Mol. Cell. Biol. 23, 2981-2990 (2003). 
42. Barbour, H., Daou, S., Hendzel, M. \& Affar, E. B. Polycomb group-mediated histone H2A monoubiquitination in epigenome regulation and nuclear processes. Nature Communications 11, 5947 (2020).

43. Zhang, X. N. et al. Transcriptome analyses reveal SR45 to be a neutral splicing regulator and a suppressor of innate immunity in Arabidopsis thaliana. BMC Genomics 18, 772 (2017).

44. Albaqami, M., Laluk, K. \& Reddy, A. S. N. The Arabidopsis splicing regulator SR45 confers salt tolerance in a splice isoform-dependent manner. Plant Mol. Biol. 100, 379-390 (2019).

45. Chen, S. L. et al. Quantitative Proteomics Reveals a Role for SERINE/ARGININE-Rich 45 in Regulating RNA Metabolism and Modulating Transcriptional Suppression via the ASAP Complex in Arabidopsis thaliana. Front. Plant Sci. 10, 1116 (2019).

46. $\mathrm{Bi}$, Y. et al. Arabidopsis ACINUS is O-glycosylated and regulates transcription and alternative splicing of regulators of reproductive transitions. Nat. Commun. 12, 945 (2021).

47. Chhun, T. et al. HSI2 repressor recruits MED13 and HDA6 to down-regulate seed maturation gene expression directly during Arabidopsis early seedling growth. Plant Cell Physiol. 57, 1689-1706 (2016).

48. Zeng, X. et al. HISTONE DEACETYLASE 9 functions with Polycomb silencing to repress FLOWERING LOCUS C expression. Plant Physiol. 182, 555-565 (2020).

49. Csorba, T., Questa, J. I., Sun, Q. \& Dean, C. Antisense COOLAIR mediates the coordinated switching of chromatin states at FLC during vernalization. Proc. Natl. Acad. Sci. U. S. A. 111, 16160-16165 (2014).

50. Li, P., Tao, Z. \& Dean, C. Phenotypic evolution through variation in splicing of the noncoding RNA COOLAIR. Genes Dev. 29, 696-701 (2015).

51. Qin, F. et al. Arabidopsis DREB2A-interacting proteins function as RING E3 ligases and negatively regulate plant drought stress-responsive gene expression. Plant Cell 20, 1693-1707 (2008).

52. Rappsilber, J., Mann, M. \& Ishihama, Y. Protocol for micro-purification, enrichment, prefractionation and storage of peptides for proteomics using StageTips. Nat. Protoc. 2, 1896 1906 (2007).

53. Mikulski, P. et al. The chromatin-associated protein PWO1 interacts with plant nuclear laminlike components to regulate nuclear size. Plant Cell 31, 1141-1154 (2019).

54. Box, M. S., Coustham, V., Dean, C. \& Mylne, J. S. Protocol: A simple phenol-based method for 96-well extraction of high quality RNA from Arabidopsis. Plant Methods 7, 7 (2011).

55. Wu, Z. et al. Quantitative regulation of FLC via coordinated transcriptional initiation and elongation. Proc. Natl. Acad. Sci. U. S. A. 113, 218-223 (2016).

56. Teves, S. S. \& Henikoff, S. Salt fractionation of nucleosomes for genome-wide profiling. Methods Mol. Biol. 833, 421-432 (2012).

57. Omidbakhshfard, M. A., Winck, F. V., Arvidsson, S., Riaño-Pachón, D. M. \& MuellerRoeber, B. A step-by-step protocol for formaldehyde-assisted isolation of regulatory elements from Arabidopsis thaliana. J. Integr. Plant Biol. 56, 527-538 (2014).

58. Nojima, T. et al. Mammalian NET-seq reveals genome-wide nascent transcription coupled to RNA processing. Cell 161, 526-540 (2015).

\section{Acknowledgements}

This work was funded by the BBSRC grant BB/P006590/1, Wellcome Trust grant 210654/Z/18/Z and supported by the BBSRC Institute Strategic Programmes GRO (BB/J004588/1) and GEN (BB/P013511/1) and a Royal Society Professorship to CD. The authors would like to thank all members of the Dean lab for discussions.

\section{Author information}

Affiliations

Cell and Developmental Biology, John Innes Centre, Norwich, UK

Pawel Mikulski, Philip Wolff, Tiancong Lu, Danling Zhu \& Caroline Dean

Present address: SUSTech-PKU Institute of Plant and Food Science, Department of Biology, Southern University of Science and Technology, Shenzhen, China 
Danling Zhu

543 Present address: State Key Laboratory of Plant Genomics and National Center for Plant Gene

544 Research, Institute of Genetics and Developmental Biology, Chinese Academy of Sciences,

545 Beijing, China

546 Tiancong Lu

547 Present address: Department of Biochemistry, University of Oxford, Oxford, UK

548 Pawel Mikulski

549 Contributions

550 PM, PW and CD conceived the study. PM performed: yeast-two-hybrid, FAIRE, nucleosome mobility, ChIPs and expression analyses in vall and PRC1 mutants. PW performed: expression analyses, histone marks and PolII ChIPs for ASAP mutants. DZ performed nucleosome position experiment. TL performed protein co-immunoprecipitation with mass spectrometry. PM, PW and CD designed the experiments and analyzed the data. CD lead the general concept of research. PW wrote the paper together with $\mathrm{CD}$ with all authors contributing to different relevant sections of the

\section{Ethics declarations}

558 Competing interest

559 The authors declare no competing interest.

\section{Additional information}

561 Supplementary Information is available for this paper. Correspondence and requests for materials

562 should be addressed to CD: caroline.dean@jic.ac.uk. Reprints and permissions information is available at www.nature.com/reprints.

\section{Figure legends}

566 Fig.1. asap mutants affect pre-cold FLC expression only. a. FLC downregulation slope during vernalization (fold change to NV). Values were normalized as relative expression to UBC. $\mathrm{N}=3$ biological replicates, error bars = propagated SEM. b. FLC expression in ASAP single mutants and different double mutant combinations within ASAP or with VAL1. Values correspond to mean relative expression to UBC. Statistics are calculated with two-tail Student T-test in comparison to wildtype $(*=\mathrm{p} \leq 0.05 ; * *=\mathrm{p} \leq 0.01 ; * * *=\mathrm{p} \leq 0.001) . \mathrm{N}=3$ biological replicates; error bars = SEM. c. Yeast-two-hybrid of the VAL1-SAP18 interaction. Yeast growth on selective medium SD-LWH is shown. Top panel corresponds to decreasing yeast culture concentration with dilutions: 1/1, 1/5. 1/25 from $\mathrm{OD} 600=0.8 . \mathrm{AD}=$ pGAD vector backbone with Gal4 activating domain $\mathrm{BD}=$ pGBKT vector backbone with Gal4 binding domain; "empty" = empty vector without insertion as negative control. Red frame depicts protein pairs showing yeast growth over negative controls. d. Schematic summary of interaction from a. e. Chromatin-bound FLC RNA enrichment over the locus. Shown as a fold change to wildtype. X-axis labels indicate midpoint of the amplicon relative to TSS and its position in FLC locus. $\mathrm{N}=3$ biological replicates, error bars = propagated SEM.

580 Fig.2. SR45 co-transcriptionally represses $\boldsymbol{F L C}$. a. RNA Polymerase II ChIP enrichment. X-axis depicts the midpoint position of amplicon at the FLC locus, $0=\mathrm{TSS}$. Y-axis corresponds to $\%$ recovery to input values normalized to housekeeping gene $A C T 7 . \mathrm{NV}=$ non-vernalized; $\mathrm{n}=3$ biological replicates; error bars $=$ SEM. b. SR45-dependent change of active-repressive histone marks. X-axis depicts midpoint position of amplicon at the $F L C$ locus. Y-axis corresponds to \% recovery to input values normalized to $\mathrm{H} 3$ enrichment. $\mathrm{NV}=$ non-vernalized; $\mathrm{n}=3$ biological replicates; error bars $=$ SEM.

587 Fig.3. FLC chromatin regulation by VAL1. a. Chromatin-bound FLC RNA enrichment over FLC locus. Shown as a fold change to wildtype. $\mathrm{X}$-axis labels indicate midpoint of the amplicon relative to TSS and its position in FLC locus. $\mathrm{N}=3$ biological replicates, error bars = propagated SEM. b. Low 
590 salt-extractable nucleosome fraction over FLC. Shown as \% recovery to uncut DNA and fold to AT4G07700 (Gypsy-like transposon). X-axis labels indicate beginning of amplicon relative to TSS, lines below axis' label indicate regions of increased low-salt nucleosome extractability in vall shown in $\mathrm{b} . \mathrm{N}=3$ biological replicates; error bars $=\mathrm{SEM}$; asterisk $=\mathrm{p}<0.05$ from two-tail Student T-test in comparison wildtype vs vall; n.s. = non-significant; $\mathrm{NV}=$ non-vernalized. c. Nucleosome position at FLC in wildtype. Results from MNase-qPCR assay shown as \% to uncut DNA. X-axis indicate beginning of amplicon relative to TSS. $\mathrm{N}=2$ biological replicates, error bars $=$ SEM. $\mathbf{d}$. FAIRE enrichment at FLC. Shown as \% recovery to non-crosslinked sample (UN-FAIRE) and fold to UBC9. $\mathrm{X}$-axis labels indicate beginning of amplicon relative to TSS. $\mathrm{N}=2$ biological replicates; error bars $=$ SEM; NV = non-vernalized.

Fig.4. H2Aub dynamics at $\boldsymbol{F L C}$. a. H2Aub chromatin -IP showing strong enrichment of H2AUb at the 5 , end of $F L C$ in ColFRI correlating with increased transcription in non-vernalized (NV) conditions. $\mathrm{X}$-axis show midpoint amplicon at $F L C . \mathrm{N}=3$ biological replicates; error bars = SEM; $\mathrm{NoAb}=$ no antibody negative control. FLC expression in wildtype backgrounds in NV is depicted in graph on the right. $\mathrm{N}=3$ biological replicates, error bars = SEM. b. H2Aub chromatin -IP correlating cold-induced decrease in FLC expression with decreased H2AUb at the 5' end of FLC. X-axis show midpoint amplicon at $F L C$. Graph on the right shows unspliced $F L C$ expression during vernalization timecourse. $\mathrm{NV}=$ non-vernalized; Nominal- $\mathrm{W}=$ number of weeks of cold; $\mathrm{N}=3$ biological replicates; error bars $=\mathrm{SEM} ; \mathrm{NoAb}=$ no antibody negative control. c. Histone mark behaviour postcold. H3K27me3 and H2Aub enrichment over FLC. X-axis show midpoint amplicon at FLC. $\mathrm{N}=3$ biological replicates; error bars $=\mathrm{SEM} ; 6 \mathrm{~W}=6$ weeks of cold treatment; $\mathrm{T}$-nominal $=$ number of days post-cold; NoAb $=$ no antibody negative control.

Fig.5. VAL1, BMI1B and NDX effect on FLC expression. a. FLC expression in mutants of VAL1, PRC1 and NDX for FLC RNA. Values correspond to mean relative expression to geometric mean of UBC and PP2A. Statistics were calculated with two-tail Student t-test in comparison to wildtype. $\mathrm{N}=$ 3 biological replicates; error bars $=$ SEM. b. $F L C$ expression level. Represented as relative expression to housekeeping genes (see methods). Statistics represent two-tail Student t-test in comparison to the wildtype, $\mathrm{N}=3$ biological replicates, error bars $=\mathrm{SEM}, \mathrm{NV}=$ non-vernalized, $\mathrm{W}=$ number of weeks of cold. c. The same data as in b. presented as FLC downregulation slope during vernalization (fold change to NV). d. H2Aub enrichment at FLC in wildtype and vall bmilB. $\mathrm{N}=3$ biological replicates; error bars $=\mathrm{SEM} ; \mathrm{NoAb}=$ no antibody negative control. co-IP. Numbers corresponds to unique peptides. IP-numeral $=$ biological replicate; $\mathrm{NV}=$ nonvernalized; Numeral-W = number of weeks of cold; T-numeral = number of days post-cold; $(-)=$ negative control. b. Yeast-2-hybrid results for PRC1-NDX interaction. Yeast growth on selective medium (-LWH) is shown. Top panel corresponds to decreasing yeast culture concentration. $\mathrm{AD}=$ pGAD vector backbone with Gal4 activating domain; $\mathrm{BD}=$ pGBKT vector backbone with Gal4 binding domain; "empty" = empty vector without insertion as negative control. Red frame depicts protein pairs showing yeast growth over negative controls (pairs with empty vector). precipitated with anti-HA antibody. c. Schematic summary of interactions from a. and b. e. and f. H2Aub ChIP results. $\mathrm{X}$-axis represents midpoint of the amplicons over $F L C$. NoAb $=$ no antibody negative control; $\mathrm{N}=3$ biological replicates for H2Aub IPs and 3 qPCR technical replicates for NoAb controls; error bars = SEM.

634 Fig.7 Functional relationship of ASAP, PRC1 and PRC2 in FLC regulation. a. ASAP and PRC1 act as co-repressors that regulate $F L C$ chromatin features and downregulate $F L C$ expression in warm. H2Aub and H3K27me3 enrichment gradually increases along with cold duration. In post-cold, 
638 H3K27me3 spreads to FLC gene body, whereas H2Aub remains localized to the FLC PRE. c. At the 639 later post-cold timepoints, $\mathrm{H} 3 \mathrm{~K} 27 \mathrm{me} 3$ is stably maintained over the $F L C$ gene body whereas $\mathrm{H} 2 \mathrm{Aub}$ 640 is removed from the chromatin. H2K27me3 thus provides long-term epigenetic silencing, whereas 641 H2Aub is a transient repression signal.

642

643 


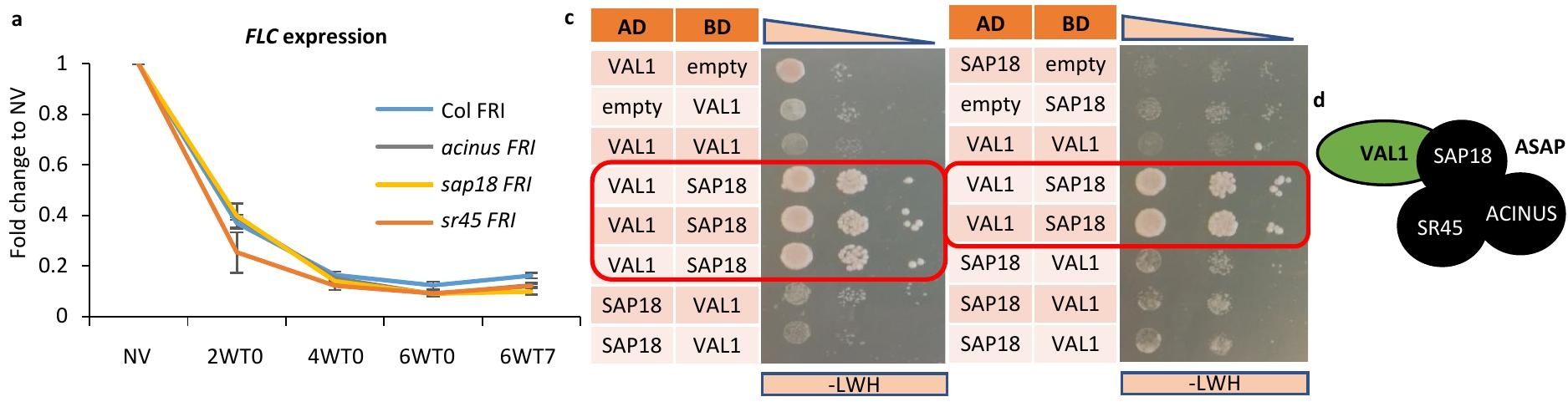

b

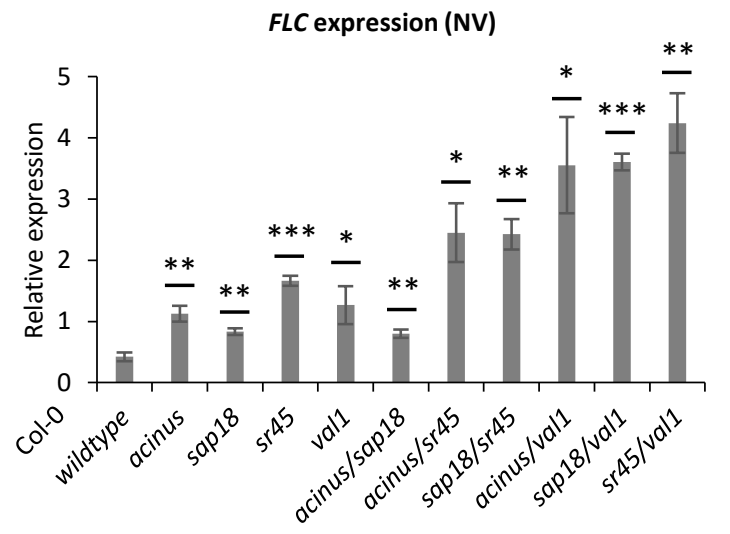

e

Chromatin-bound RNA enrichment (NV)

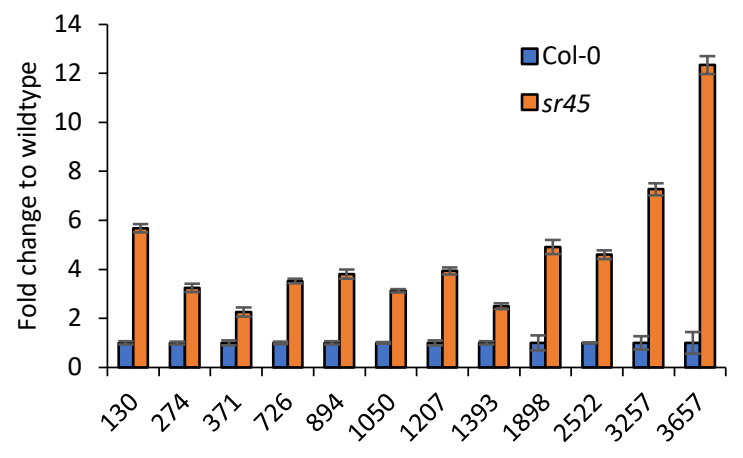

Fig.1. asap mutants affect pre-cold $F L C$ expression only. a. FLC downregulation slope during vernalization (fold change to NV). Values were normalized as relative expression to UBC. $\mathrm{N}=3$ biological replicates, error bars = propagated SEM. b. $F L C$ expression in ASAP single mutants and different double mutant combinations within ASAP or with VAL1. Values correspond to mean relative expression to UBC. Statistics are calculated with two-tail Student T-test in comparison to wildtype $(*=\mathrm{p} \leq 0.05 ; * *=\mathrm{p} \leq 0.01 ; * * *=\mathrm{p} \leq 0.001) . \mathrm{N}=3$ biological replicates; error bars = SEM. c. Yeast-two-hybrid of the VAL1-SAP18 interaction. Yeast growth on selective medium SD-LWH is shown. Top panel corresponds to decreasing yeast culture concentration with dilutions: 1/1, 1/5. 1/25 from $\mathrm{OD} 600=0.8 . \mathrm{AD}=$ pGAD vector backbone with Gal4 activating domain; $\mathrm{BD}=$ pGBKT vector backbone with Gal4 binding domain; "empty" = empty vector without insertion as negative control. Red frame depicts protein pairs showing yeast growth over negative controls. d. Schematic summary of interaction from a. e. Chromatin-bound FLC RNA enrichment over the locus. Shown as a fold change to wildtype. X-axis labels indicate midpoint of the amplicon relative to TSS and its position in $F L C$ locus. $\mathrm{N}=3$ biological replicates, error bars = propagated SEM. 


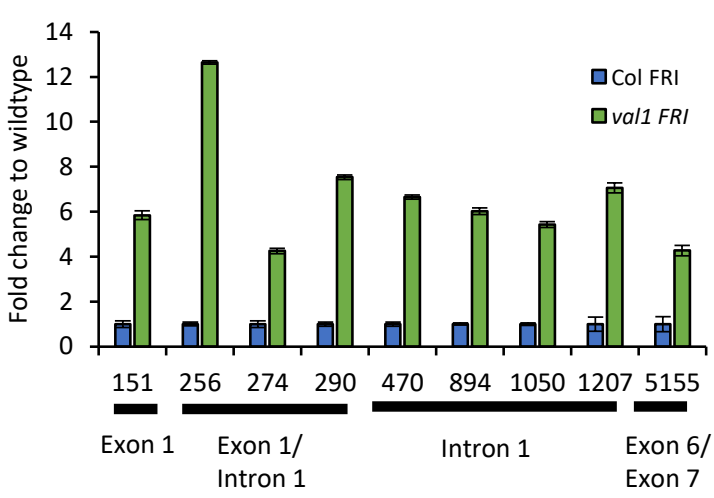

C

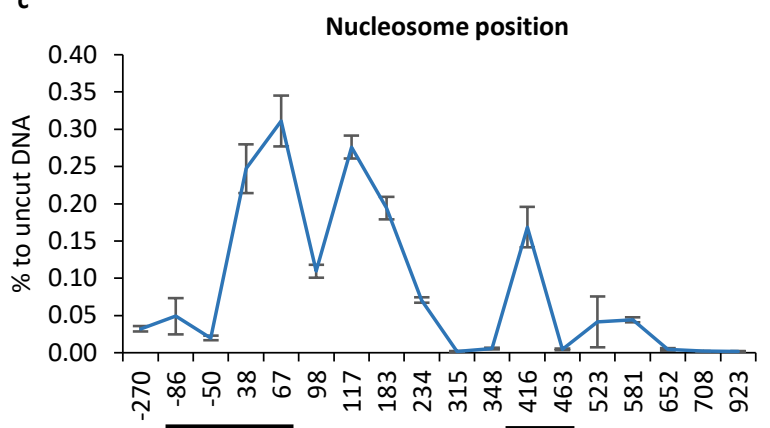

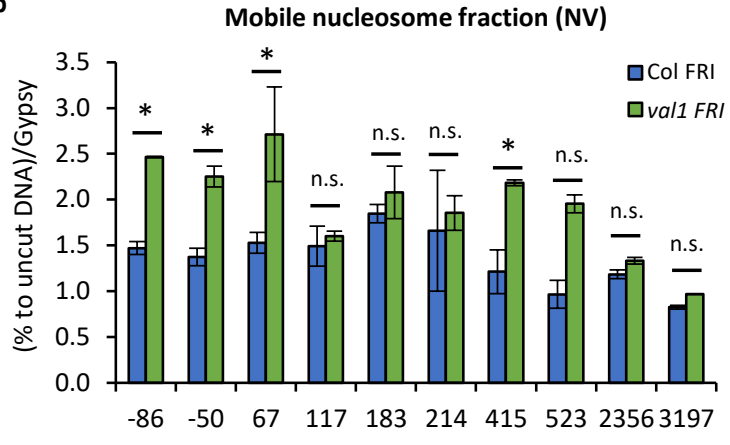

d

FAIRE enrichment (NV)

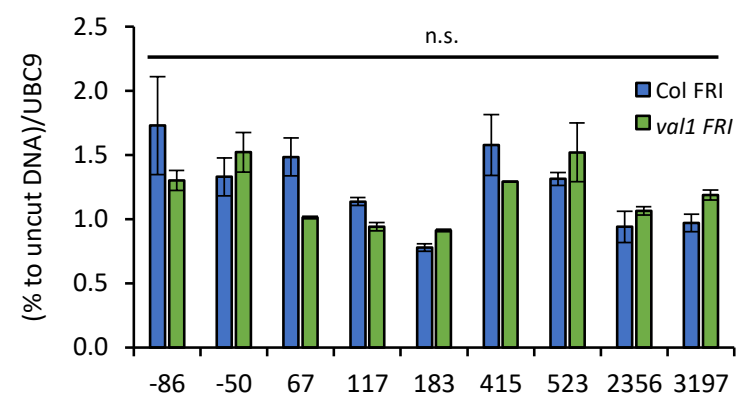

Fig.3. FLC chromatin regulation by VAL1. a. Chromatin-bound FLC RNA enrichment over FLC locus. Shown as a fold change to wildtype. X-axis labels indicate midpoint of the amplicon relative to TSS and its position in FLC locus. $\mathrm{N}=3$ biological replicates, error bars = propagated SEM. $\mathbf{b}$. Low salt-extractable nucleosome fraction over FLC. Shown as \% recovery to uncut DNA and fold to AT4G07700 (Gypsy-like transposon). X-axis labels indicate beginning of amplicon relative to TSS, lines below axis' label indicate regions of increased low-salt nucleosome extractability in vall shown in $\mathrm{b} . \mathrm{N}=3$ biological replicates; error bars = SEM; asterisk $=\mathrm{p}<0.05$ from two-tail Student T-test in comparison wildtype vs vall; n.s. = non-significant; NV = non-vernalized. $\mathbf{c}$. Nucleosome position at $F L C$ in wildtype. Results from MNase-qPCR assay shown as \% to uncut DNA. X-axis indicate beginning of amplicon relative to TSS. N = 2 biological replicates, error bars = SEM. d. FAIRE enrichment at FLC. Shown as \% recovery to non-crosslinked sample (UN-FAIRE) and fold to UBC9. X-axis labels indicate beginning of amplicon relative to TSS. $\mathrm{N}=2$ biological replicates; error bars = SEM; NV = non-vernalized. 


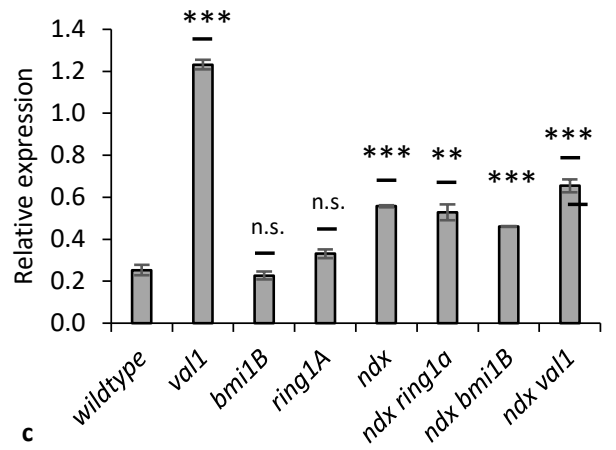

FLC expression

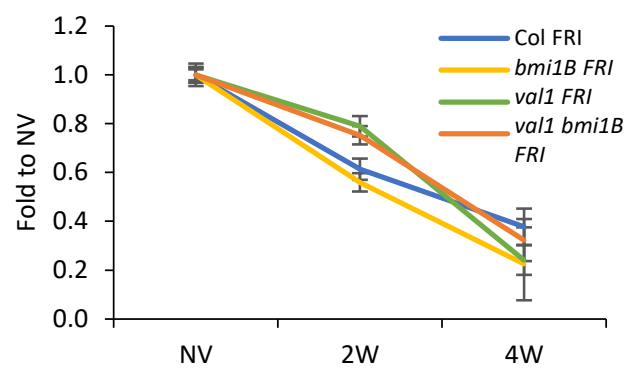

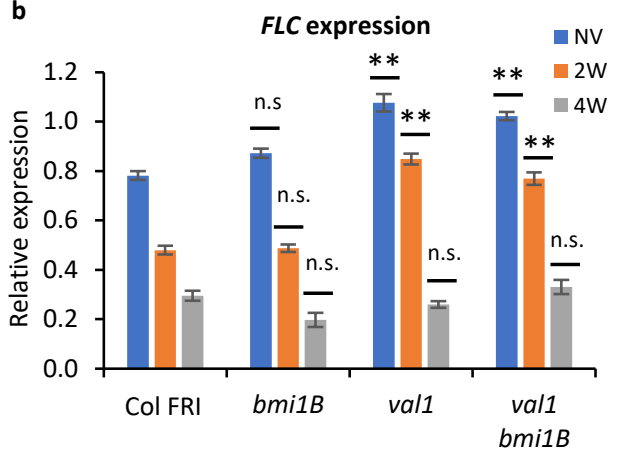

d

\section{H2Aub ChIP (NV)}

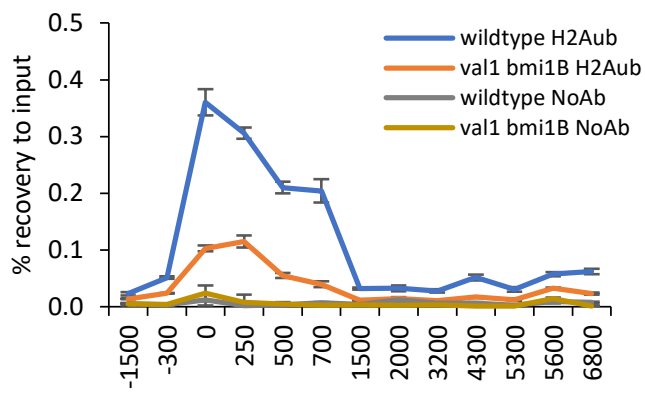

Fig.5. VAL1, BMI1B and NDX effect on FLC expression. a. FLC expression in mutants of VAL1, PRC1 and NDX for FLC RNA. Values correspond to mean relative expression to geometric mean of UBC and PP2A. Statistics were calculated with two-tail Student t-test in comparison to wildtype. $\mathrm{N}=3$ biological replicates; error bars = SEM. b. $F L C$ expression level. Represented as relative expression to housekeeping genes (see methods). Statistics represent two-tail Student t-test in comparison to the wildtype, $\mathrm{N}=3$ biological replicates, error bars $=\mathrm{SEM}, \mathrm{NV}=$ non-vernalized, $\mathrm{W}=$ number of weeks of cold. $\mathbf{c}$. The same data as in $\mathbf{b}$. presented as FLC downregulation slope during vernalization (fold change to NV). d. H2Aub enrichment at $F L C$ in wildtype and vall bmilB. $\mathrm{N}=3$ biological replicates; error bars $=\mathrm{SEM} ; \mathrm{NoAb}=$ no antibody negative control. 


\begin{tabular}{|c|c|c|c|c|c|c|c|c|c|c|c|}
\hline \multirow{2}{*}{ Locus } & \multirow{2}{*}{ Uniprot ID } & \multirow{2}{*}{ Name } & IP1 (-) & IP2 (-) & IP1 (-) & IP2 (-) & IP1 & IP1 & IP2 & IP1 & IP2 \\
\cline { 4 - 12 } & & & NV & NV & 4WT0 & 4WT0 & NV & 4WT0 & 4WT0 & 4WT7 & 4WT7 \\
\hline AT2G30470 & Q8W4L5 & VAL1 & 0 & 0 & 0 & 0 & 53 & 69 & 80 & 61 & 66 \\
AT1G06770 & Q9M9Y4 & BMI1B & 0 & 0 & 0 & 0 & 3 & 7 & 13 & 4 & 6 \\
AT2G30580 & Q94AY3 & BMI1A & 0 & 0 & 0 & 0 & 10 & 14 & 12 & 11 & 7 \\
AT5G44280 & Q9FKW0 & RING1A & 0 & 0 & 0 & 0 & 6 & 6 & 5 & 4 & 3 \\
AT4G03090 & F4JI44 & NDX & 0 & 0 & 0 & 0 & 3 & 8 & 13 & 7 & 9 \\
\hline
\end{tabular}

d

\section{H2Aub ChIP (NV)}

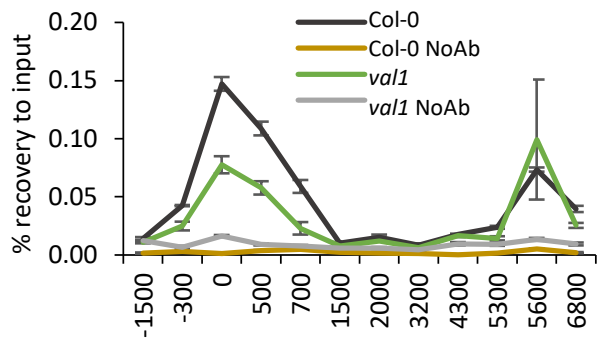

H2Aub ChIP (NV)

e
H2Aub ChIP (NV)

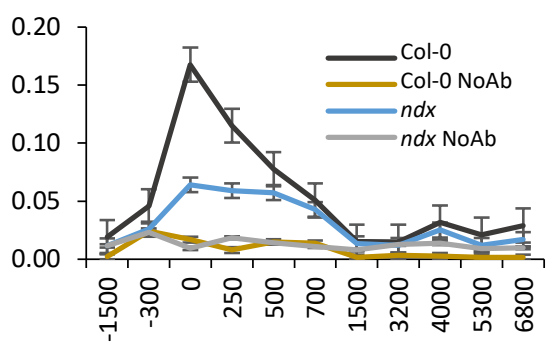

H2Aub ChIP (6W cold)

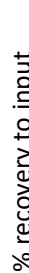

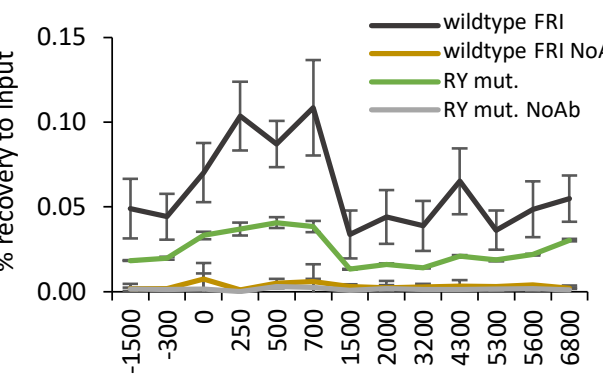

b

Non

PA

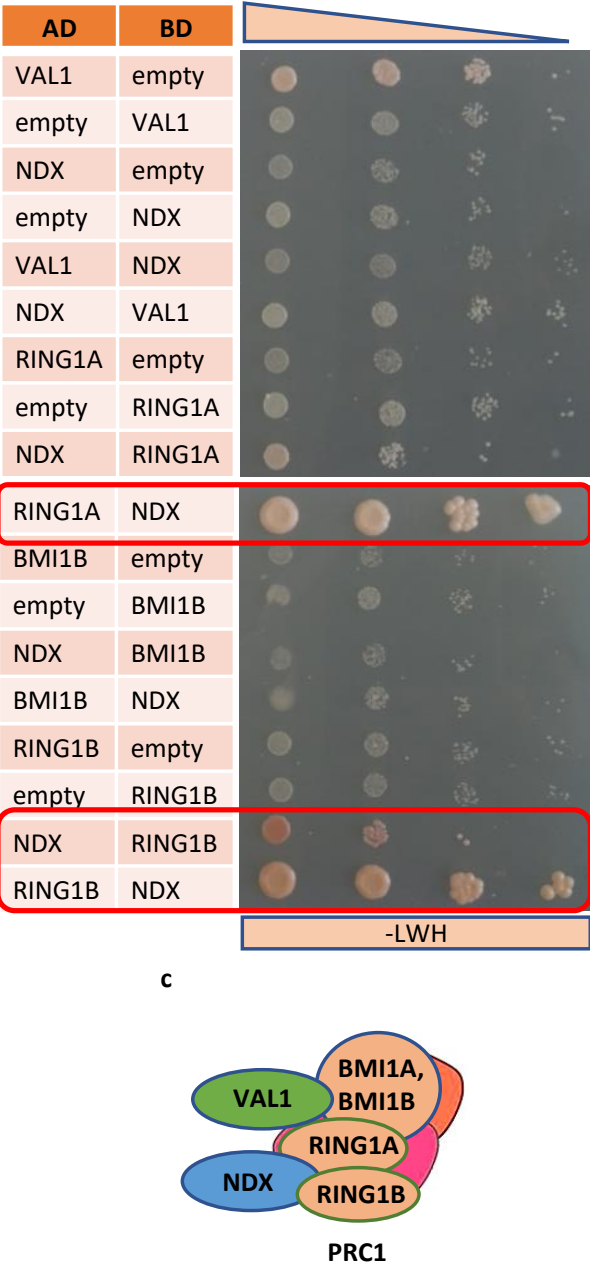

Fig.6. VAL1, PRC1 and NDX physically interact and are required for H2Aub accumulation at the FLC nucleation region. a. IP-MS/MS results for PRC1 and NDX from crosslinked VAL1-HA co-IP. Numbers corresponds to unique peptides. IP-numeral = biological replicate; NV = non-vernalized; Numeral-W = number of weeks of cold; T-numeral = number of days post-cold; $(-)$ = negative control. b. Yeast-2-hybrid results for PRC1-NDX interaction. Yeast growth on selective medium (-LWH) is shown. Top panel corresponds to decreasing yeast culture concentration. AD = pGAD vector backbone with Gal4 activating domain; $\mathrm{BD}=$ pGBKT vector backbone with Gal4 binding domain; "empty" = empty vector without insertion as negative control. Red frame depicts protein pairs showing yeast growth over negative controls (pairs with empty vector). precipitated with anti-HA antibody. c. Schematic summary of interactions from a. and b. e. and f. $\mathrm{H} 2 \mathrm{Aub}$ ChIP results. X-axis represents midpoint of the amplicons over $F L C$. NoAb $=$ no antibody negative control; $\mathrm{N}=3$ biological replicates for H2Aub IPs and 3 qPCR technical replicates for NoAb controls; error bars $=$ SEM. 
